\title{
DNA RNA and Peptide Synthesis Shared Resource
}

National Cancer Institute

\section{Source}

National Cancer Institute. DNA RNA and Peptide Synthesis Shared Resource. NCI

Thesaurus. Code C39378.

DNA, RNA and Peptide Synthesis Laboratories provide support to researchers regarding oligonucleotide and peptide chemistry. Primary services of this facility include: synthesis of DNA, RNA, chimeric DNA/RNA molecules, and custom oligonucleotides, including those containing modified bases, modified sug ars, and incorporated labels and linkers, and synthesis of various types of peptides. 